\title{
A Method for Sudanese Vehicle License Plates Detection and Extraction
}

\author{
Musab Bagabir, \\ College of Computer Science and Information \\ Technology, \\ Sudan University for Science and Technology, \\ Khartoum, Sudan
}

\author{
Mohammed Elhafiz \\ College of Computer Science and Information \\ Technology, \\ Sudan University for Science and Technology, \\ Khartoum, Sudan
}

\begin{abstract}
License Plate Detection and Extraction is an important phase of Vehicle License Plate Recognition systems, which has been an active research topic in the computer vision domain in order to identify vehicles by their license plates without direct human intervention. This paper presents a simple, fast and automatic License Plate Detection method for the current shape of Sudanese license plate. The proposed method involves several steps: green channel extraction, edge detection, regions of interest selection, dilation operation with especial structural element and connected component analysis. In order to analyze the performance and efficiency of the proposed method a data set for Sudanese vehicles has been created. Using this new data set, number of experiments has been carried out. Comparing with other countries license plate detection the achieved results is satisfactory.
\end{abstract}

Keywords: Green Channel Extraction; Edge Detection; Morphological Operation; Connected Component Analysis

\section{INTRODUCTION}

Vehicle License Plate Recognition (VLPR) systems is an important component for automating many control and surveillance systems, such as road traffic monitoring, parking lots, access control, highway electronic toll collection, red light violation enforcement, finding stolen cars and gathering traffic flow statistics [1]. Due to the differences of license plates in formats, styles, colors and size from one country to another, the field of VLPR and its applications has attracted many researchers in many countries to search and develop systems that solve these different problems. Therefore, so far, many methods have been proposed for VLPR depending on the country's license plate characteristics.

License Plate Detection (LPD) has been considered as the most important and essential phase of VLPR systems, which is directly influences the success and the accuracy of VLPR systems [3, 4]. For that, LPD requires more attention; moreover detecting a license plate on a complex background is a difficult task. Thus there are many factors should be considered in order to successfully detect and extract the license plate, for example: image quality, different plate sizes and designs, plate location and Background details and complexity [3].

According to Sarfraz et al the license plate extracted from the gray-scaled image by detecting vertical edges using Sobel edge detector, which uses a $3 \times 3$ mask, then filtering out unwanted regions by applying seed-filling algorithm [5]. The license plate region extracted by comparing the size ratio of the rectangular area between two vertical regions with the actual standard size ratio of the license plate. Alginahi uses the method reported by Sarfraz et al without filtering step in order to locate different License Plate types in shapes and size [6]. But Basalamah works depends on finding the black cross that centers Saudi Arabian plate, so an edge detector is applied to find the horizontal and vertical maps, then before median filter performed, the binary image is obtained by using the average value of pixels in each map as a threshold [7].
Abulgasem et al proposed Radial Basis Function Neural Network (RBF NN) to detect the Libyan license plate. First sobel edge detector applied, and then some morphological methods is used to thicken edges and remove unwanted edges. The remaining regions are detected and categorized into "plate" and "not plate" manually to train the $\mathrm{RBF} N \mathrm{~N}$, which afterwards is used to detect the license plate automatically in other images during the testing phase [8].

Mousa presented an algorithm for Palestine LPD based on canny edge detector. This edge detection method is used to find image's edges based on local maxima of the gradient, which calculated by the derivative of a Gaussian filter [9]. Rasheed et al use canny edge detection operator and Hough lines to detect and extract the license plate [10].

The method proposed by Mohammad et al for LPD based on identifying the location of screws that hold the plates in place using pattern matching, plate aspect ratio (width to height ratio), and intensity levels. Then by applying coordinate system, the plate area is masked with respect to each screw position [11]

Shidore et al proposed LPD technique for Indian vehicles by using Sobel filter, morphological operations and connected component analysis [12]. Indian LPD mechanism developed by Davis et al, in which the gray-scaled image is converted to binary image by using adaptive thresholding. Then applied unwanted lines elimination algorithm based on $3 \times 3$ mask, which is moved throughout the image to identify the central pixel and testing the remaining 8 neighbor pixels. After that the vertical edges is extracted fallowed by highlighting the required regions technique. Once again unwanted lines elimination algorithm is used. As last step, the image is scanned for continuous black pixel in order to obtain the two diagonal corners of the license plate [13].

Deb et al proposed Sliding Concentric Window (SCW) based system to detect Korean license plate. After applying SCW on vehicle image, HSI color model is used for candidate region 
color verification witch based on hue and intensity in HSI color model [14].

The remainder of this paper is structured as follows: Section 2, introduces the Sudanese vehicle license plate. Section 3, describes the proposed method. The experimental results are provided along with discussion in section 4 . Section 5 concludes the paper.

\section{SUDANESE CAR LICENSE PLATE}

The Sudanese vehicles license plates are categorized in a number of types [2], that categorization was based on the differences of plates background color and characters color, Table 1. gives some information about these types.

Table 1. Sudanese Vehicles Plates Types

\begin{tabular}{|c|c|c|}
\hline Type & $\begin{array}{c}\text { Background } \\
\text { Color }\end{array}$ & $\begin{array}{c}\text { Characters } \\
\text { Color }\end{array}$ \\
\hline Private vehicles & White & Black \\
\hline $\begin{array}{c}\text { Commercials } \\
\text { (Passenger) }\end{array}$ & White & Green \\
\hline $\begin{array}{c}\text { Commercials } \\
\text { (Goods) }\end{array}$ & Black & White \\
\hline Police & Blue & White \\
\hline Government & Yellow & Black \\
\hline
\end{tabular}

The size of all plate types is $32 \times 16$ centimeters (see Figure 1 ). The plate has been divided into three regions; one region at the top part of the plate, which contains the name of the country "SUDAN" written in English and Arabic. The other two regions at the bottom part of the plate. They were separated by a silver metallic bar (Old Plates) Figure 1(a), or a vertical text “جمهورية السودان" (New Plates) Figure 2(b).The right bottom part consists of numerals (1 to 5 numbers) written in English and Arabic. Where the left bottom part consists of characters or a character and number written in English and Arabic, the characters are an abbreviation of Sudan states names, and the number to keep the sequence of the numbering. This study will focuses on the first type: private vehicles, as shown in Figure 1 (a) and (b).

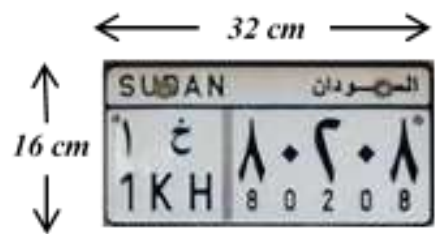

(a)

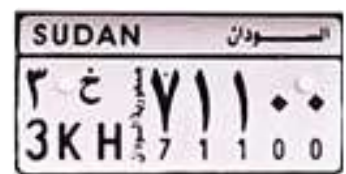

(b)

Figure 1. Sudanese License Plate (a) Plate with Silver Metallic Bar (b) Plate with Vertical Text

\section{THE PROPOSED METHOD}

The proposed method designed for Sudanese vehicle LPD. It composed of four of stages, including green channel and edge detecting, region of interest filtration, dilation and candidate regions detection and accurate plate detection/extraction, as shown in Figure 2.

The input of the method is the original image of the vehicle in RGB scale of size $2048 \times 1536$ pixels taken from real scene. The details of other stages are presented in the following subsections.

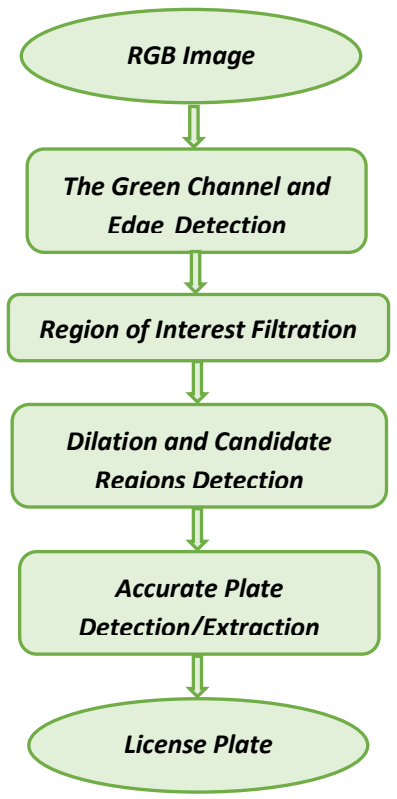

Figure 2. The Proposed Method

\subsection{The Green Channel and Edge Detection}

The RGB image consists of three channels red, green and blue, the value of each channel in the range 0-256, whereas the gray scale image contains only one channel [15]. Thus, extraction of one channel will decrease the computational time, as well as the storage space. Experiments showed that the green channel provides sufficient contrast for the image, which in turn directly increases the efficiency of the proposed method than other channels or gray conversion. Thus, green channel extracted as in Figure 3. Afterwards, median filter is applied to remove noises like random occurrences of black and white pixels.

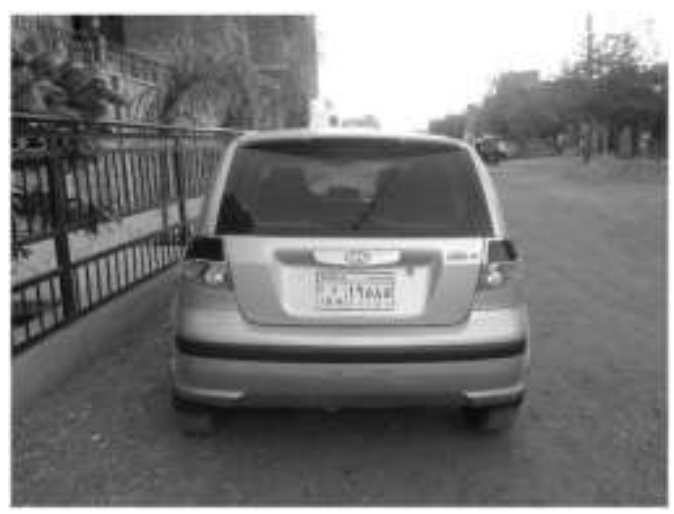

Figure 3. Green Channel Extraction 
The next step is edge detection. Edges in images are areas with strong intensity contrasts, which represent a boundary between different regions. Detecting the edges of an image significantly reduces the amount of data and it helps in filtering out the useless information.

Primary investigation of this research shows that sobel edge detection has better results on Sudanese vehicles data set. The Sobel edge detector applies a $3 \times 3$ mask on the input image and gives the resultant binary image (see Figure 4(a)). Then, dilation operation with disk structural element is performed to thicken the edges, that is due to; edge's lines do not completely cover the region of interest. Figure 4(b) shows the resultant image.

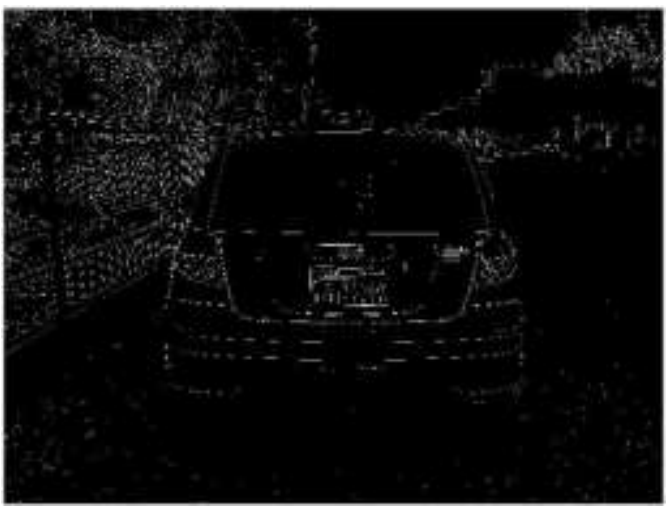

(a)

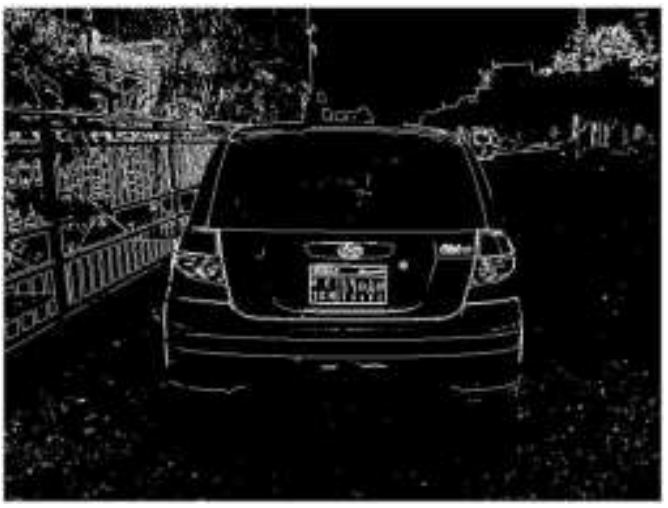

(b)

Figure 4. (a) Edge Detection (b) Thicken Edge

\subsection{Region of Interest Filtration}

Filtration process is performed either to select regions that satisfy some particular features or eliminates unwanted regions on the image [16]. Filtration get the main aim of this step done, which is to obtain a filtered image has as possible all license plate contents except its boundaries. The step begins with removing the very small regions based on the number of white pixels of each one (Region Area). Afterwards, all regions in the resultant image were detected; their widths and heights used as features to select regions have a specific width and height. Although license plate contents are successfully well segmented, but there are some regions belong to the background are also selected, as shown in Figure 5 (a).
Thus, those unwanted regions should be removed or reduced. This is achieved by filling each region in the image and calculating their areas. Then, the region is selected if its area is greater or equal to $A_{\min }$ and less or equal to $A_{\max }$, otherwise

the region is removed, where:

$$
\begin{aligned}
& A_{\text {min }}: \text { Minimum Region Area } \\
& A_{\text {max }}: \text { Maximum Region Area }
\end{aligned}
$$

$A_{\min }$ and $A_{\max }$ have been set during the experiments. Figure 5(b) shows the resultant image.

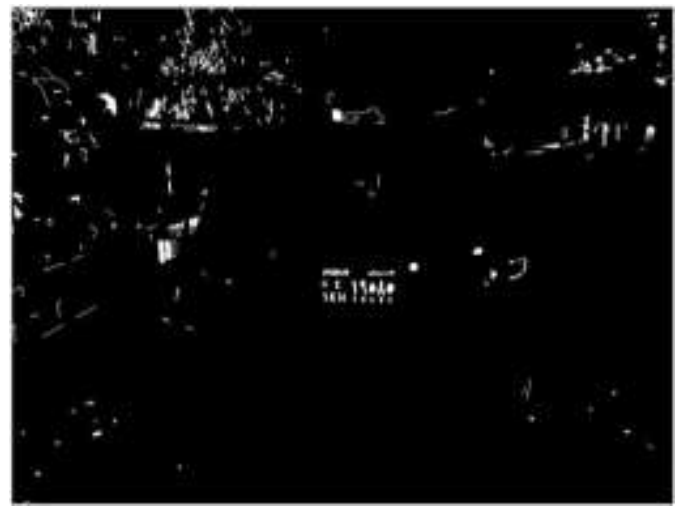

(a)

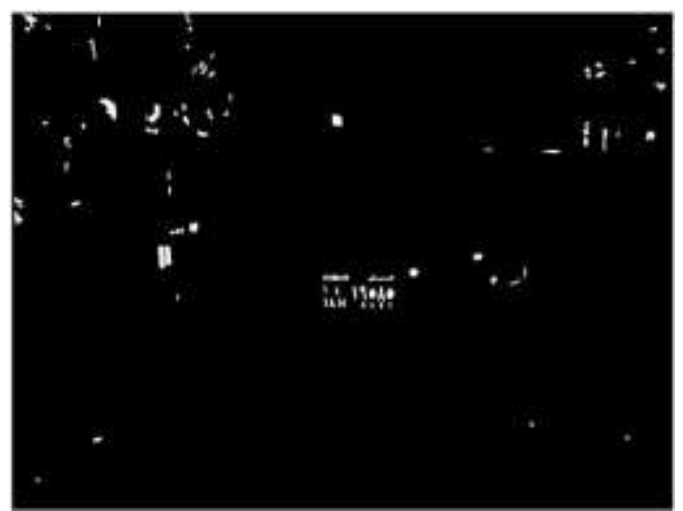

(b)

Figure 5. (a) License Plate Contents Selection (b) Remove Unwanted Regions

\subsection{Dilation and Candidate Regions Detection}

The aim of this stage is to obtain the candidate regions that might be the license plate region. It is based on the idea of merging the closed regions as well as removing unwanted regions. Therefore, the morphological operation dilation has been used with specific structuring element (SE) to expand the regions. When regions expand, the gaps reduced.

According to Sudanese car license plate layout as mentioned in section 2, the dilation operation is used three times with different SE values to merge each group of the license plate components separately. For instance, suppose that the license plate is divided into two parts vertically from the metallic bar, and then each part (Left/Right) contains three rows of characters as illustrated in Figure 6. 


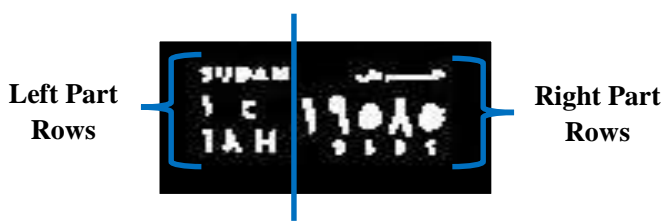

Figure 6. Visualization of How the Plate Vertically Divided

First performing dilation with SE value of size $30 \times 15$ pixel to merge the three rows of characters of each left and right part separately. Then removing any object contiguous to the border of the resultant image and others objects those their area less than the expected area of merged each two parts as in Figure 7(a). The purpose of second and third dilation is to join the two parts horizontally. This is achieved by using a special single row-SE to restrict the expansion of objects/regions along the horizontal direction only. After the second dilation, the bigger objects than a specific area value were selected, and then third dilation performed as shown in Figure 7(b).

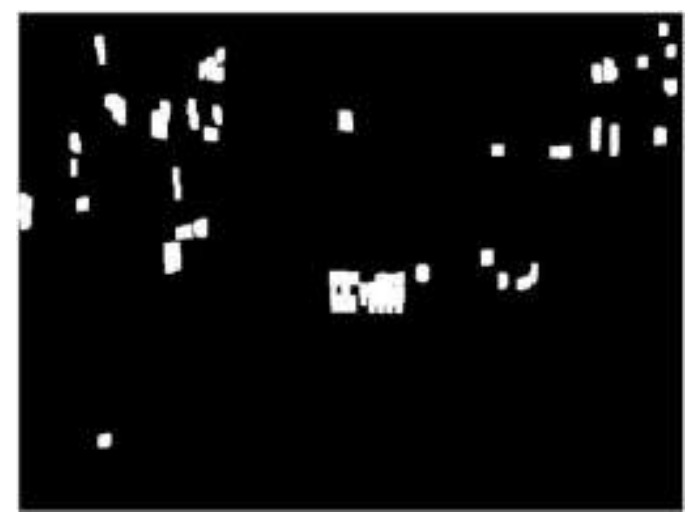

(a)

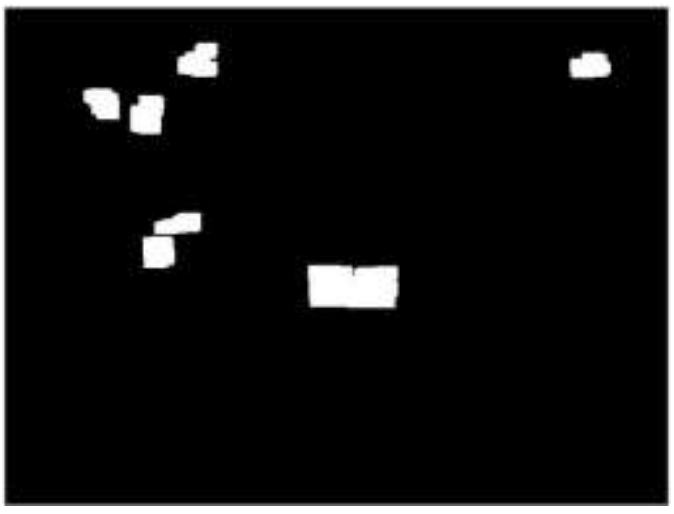

(b)

Figure 7. (a) First Dilation. (b) Second and Third Dilation

\subsection{Accurate Plate Detection/Extraction}

The license plate detection and extraction stage identifies the accurate region of the license plate. The connected components (objects/regions) analysis is performed to identify each object/region in the resultant binary image from previous stage. For each connected component in the image, some features are calculated in order to identify the license plate region. While each connected component surrounded by smallest rectangle as illustrated in Figure 8, those features are explained as follows:

a. Height to width ratio (aspect ratio).

b. The rectangle area (height $\times$ width).

c. The possible number of white pixels in the rectangle.

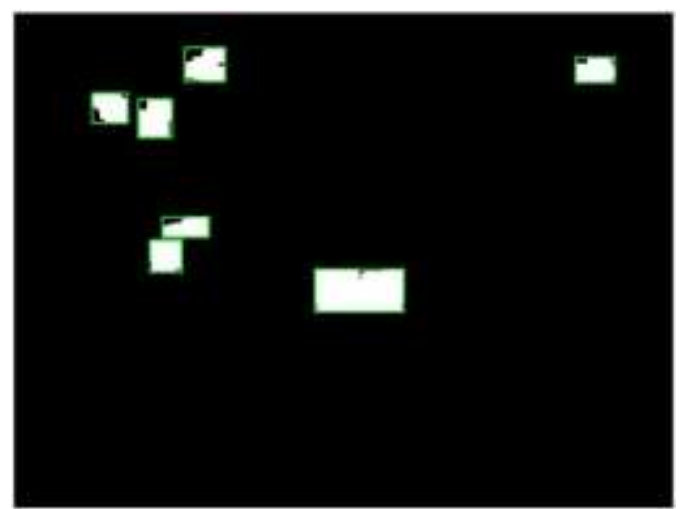

Figure 8. Connected Component are Surrounded by Rectangle

Afterwards, the accurate plate region is detected if the region position in the lower two thirds of the image. Figure 9 shows the result of this stage.

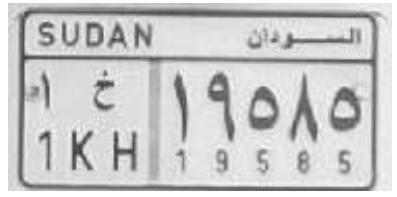

Figure 9. Extracted License Plate

\section{EXPERIMENTS AND DISCUSSIONS}

Experiments have been carried out under MATLAB R2013a (Version 8.1.0.604) environment to test the proposed method and to measure its accuracy. 200 color images with size of $2048 \times 1536$ pixels used for testing the method. Tested images have been captured in real scene, and the distance between the camera and the vehicle varied from 2 up to 3 meter.

The results of the Experiments are presented in Table 2. It shows the detection and extraction of the plate region accuracy is $98.5 \%$.

Table 2. Experiments Results

\begin{tabular}{|c|c|c|}
\hline $\begin{array}{c}\text { Total Number Of } \\
\text { Images }\end{array}$ & $\begin{array}{c}\text { Successful } \\
\text { Extraction }\end{array}$ & $\begin{array}{c}\text { Unsuccessful } \\
\text { Extraction }\end{array}$ \\
\hline 200 & 197 & 3 \\
\hline $100 \%$ & $98.5 \%$ & $1.5 \%$ \\
\hline
\end{tabular}

On the other hand, the failure in detection and extraction can be due to damaged plates, the characters color changed to white, decorative items covered the plate and the reflection of the sun on the plate region as illustrated in Table 3. 
Table 3. Failure in Detection and Extraction

\begin{tabular}{|c|c|}
\hline Failed Detection & Comments \\
\hline & $\begin{array}{c}\text { Conse Plate } \\
\text { characters color } \\
\text { changed to white }\end{array}$ \\
\hline & $\begin{array}{c}\text { License Plate } \\
\text { covered by } \\
\text { decorative items }\end{array}$ \\
\hline \\
\hline
\end{tabular}

\section{CONCLUSION}

The purpose of this paper is to presents a method for an automatic vehicle license plate detection and extraction. The proposed method is mainly designed for Sudanese license plate, according to the literature it is considered as first of its kind for Sudanese vehicle license plates.

According to the state of the art of vehicle license plate extraction, the proposed method is implemented through four stages: green channel and edge detecting, region of interest filtration, dilation and candidate regions detection and accurate plate detection/extraction.

The proposed method succeeds in detecting and extracting the plates efficiently and accurately with high rate percentage ( $98.5 \%$ for the given data set).

\section{REFERENCES}

[1] Hu, H., Zhang, Z., \& Bai, Y. (2012). Car License Plate Location Based on Mathematical Morphology. In Recent Advances in Computer Science and Information Engineering. Springer Berlin Heidelberg: 415-420.

[2] Bagabir, M., Mariyam, S., Elhafiz, M. and Ahmed, A. (2015). Multi Objective Segmentation for Vehicle License Plate Detection with Immune-based Classifier: A General
Framework. International Journal of Computer Applications Technology and Research 04 (04), 322 - 326.

[3] Du, S., Ibrahim, M., Shehata, M., \& Badawy, W. (2013). Automatic license plate recognition (ALPR): A state-ofthe-art review. Circuits and Systems for Video Technology, IEEE Transactions on, 23(2), 311-325.

[4] Patel, C., Shah, D., \& Patel, A. (2013). Automatic Number Plate Recognition system (ANPR): A survey. International Journal of Computer Applications, 69(9), 21-33.

[5] Sarfraz, M., Ahmed, M. J., \& Ghazi, S. (2003, July). Saudi Arabian License Plate Recognition system. In Geometric Modeling and Graphics, 2003. Proceedings. 2003 International Conference on (pp. 36-41). IEEE.

[6] Alginahi, Y. M. (2011). Automatic Arabic License Plate Recognition. International Journal of Computer and Electrical Engineering, 3(3), 454-460.

[7] Basalamah, S. (2013). Saudi License Plate Recognition. International Journal of Computer and Electrical Engineering, 5(1), 1.

[8] Abulgasem, N. A., Mohamad, D., \& Mohamad Hashim, S. Z. (2011). Automatic License Plate Detection and Recognition Using Radial Basis Function Neural Network. International Journal of Computer Vision and Applications (IJCVA), 1(1).

[9] Mousa, A. (2012). Canny Edge-Detection Based Vehicle Plate Recognition. International Journal of Signal Processing, Image Processing \& Pattern Recognition, $5(3)$.

[10] Rasheed, S., Naeem, A., \& Ishaq, O. (2012). Automated Number Plate Recognition using hough lines and template matching. In Proceedings of the World Congress on Engineering and Computer Science (Vol. 1, pp. 24-26).

[11] Mohammad, K., Agaian, S., \& Saleh, H. (2011, February). Practical Automatic Arabic License Plate Recognition System. In IS\&T/SPIE Electronic Imaging (pp. 78810V78810V). International Society for Optics and Photonics.

[12] Shidore, M. M., \& Narote, S. P. (2011). Number Plate Recognition for Indian Vehicles. IJCSNS, 11(2), 143.

[13] Davis, A. M., Arunvinodh, C., \& Arathy Menon, N. P. (2015, March). Automatic License Plate Detection Using Vertical Edge Detection Method. In Innovations in Information, Embedded and Communication Systems (ICIIECS), 2015 International Conference on (pp. 1-6). IEEE.

[14] Deb, K., Khan, I., Saha, A., \& Jo, K. H. (2012). An Efficient Method of Vehicle License Plate Recognition Based on Sliding Concentric Windows and Artificial Neural Network. Procedia Technology, 4, 812-819.

[15] Gonzalez, R. C., Woods, R. E., \& Eddins, S. L. (2009). Digital Image processing using MATLAB ${ }^{\circledR}$. United States: Gatesmark Publishing.

[16] Ibrahim, N. K., Kasmuri, E., Jalil, N. A., Norasikin, M. A., Salam, S., \& Nawawi, M. R. M. (2013). License Plate Recognition (LPR): A Review with Experiments for Malaysia Case Study. The International Journal of Soft Computing and Software Engineering. (JSCSE), 3(3). 